\title{
Resonant reactions of astrophysical interest studied by means of the Trojan Horse Method. Two case studies
}

\author{
Marco La Cognata ${ }^{1, *}$, Claudio Spitaleri ${ }^{1,2}$, Silvio Cherubini ${ }^{1,2}$, Marisa Gulino ${ }^{1,3}$, Livio \\ Lamia $^{1,2}$, Rosario G. Pizzone ${ }^{1}$, Stefano Romano ${ }^{1,2}$, and Aurora Tumino ${ }^{1,3}$ \\ ${ }^{1}$ Laboratori Nazionali del Sud - INFN, Catania, Italy \\ ${ }^{2}$ Dipartimento di Fisica e Astronomia, University of Catania, Catania, Italy \\ ${ }^{3}$ Facoltà di Ingegneria e Architettura, Kore University of Enna, Enna, Italy
}

\begin{abstract}
Resonant reactions in astrophysics play and important role as unexpected resonances may enhance the astrophysical factor with respect to the direct reaction contribution, altering the predicted nucleosynthesis scenarios by changing, for instance, the expected nucleosynthesis path. They also are of great interest in nuclear structure studies, since the determination of energies, spinparities and partial widths sheds light on the occurrence of cluster structures, for instance. However, nuclear reactions in most astrophysical environments usually take place at energies below about $1 \mathrm{MeV}$, leading to an exponential decrease of the cross sections due to the effect of the penetration of the Coulomb barrier. Also, at energies so low to be comparable with those associated to electronic degrees of freedom, the effect of atomic and/or molecular clouds cannot be neglected, resulting in a shielding of nuclear charges and in an enhancement of the cross sections with respect to the case of bare nuclei (the so called electron screening effect). Owing to vanishingly small cross sections and ambiguities in the extrapolation due to the electron screening, supplying accurate cross sections for astrophysical modeling is extremely challenging. Indirect methods have been introduced to explore the energy range of astrophysical interest with no need of extrapolation, even guided by theoretical arguments. In particular, the Trojan Horse Method makes use of quasi-free reactions with three particles in the exit channel, $a+A \rightarrow c+C+s$, to deduce the cross section of the reaction of astrophysical interest, $a+x \rightarrow c+C$, under the hypothesis that $A$ shows a strong $x+s$ cluster structure. Even if measurements are carried out above astrophysical energies to be free from Coulomb suppression and electron screening, the range of astrophysical interest can be covered thanks to the $x-s$ intercluster motion and binding energy. In these proceedings we will show the application of the THM, in the case of resonant reactions, using the generalised R-matrix approach introduced by A.M. Mukhamedzhanov. We will discuss the possibility to extract resonance parameters from the Trojan Horse data and perform a full spectroscopic study of low-energy and even sub-threshold resonances. In particular, we will focus on the ${ }^{19} \mathrm{~F}(p, \alpha){ }^{16} \mathrm{O}$ and the ${ }^{13} \mathrm{C}(\alpha, n){ }^{16} \mathrm{O}$ reactions, of particular importance in the case of asymptotic giant branch stars and in the synthesis of heavy elements by means of the s-process.
\end{abstract}

\footnotetext{
*e-mail: lacognata@lns.infn.it
} 


\section{Introduction}

Nuclear astrophysics deals with the investigation of nuclear physics phenomena influencing astrophysical sites such as stars or the early universe. Nuclear reactions power many astrophysical processes like energy production and synthesis of the chemical elements, therefore, cross sections $\sigma(E)$ are among the main input parameters of the codes used to model these scenarios. However, in the case of many astrophysical phenomena, such as quiescent stellar burning, energies of interest are so low that for charged particles the Coulomb barrier strongly diminishes cross sections making the measurement of such cross sections at energies of interest for astrophysics not always possible.

These energies (the so-called Gamow window [1,2]) usually vary between few keV and few hundreds of $\mathrm{keV}$, therefore cross sections can be much smaller than $1 \mathrm{nb}$, making extrapolation from high energies the only way to estimate them at the energies of interest. Extrapolation is often performed by means of the astrophysical factor [1, 2]:

$$
S(E)=\sigma(E) E \exp (2 \pi \eta),
$$

where $\eta=\mathrm{Z}_{1} \mathrm{Z}_{2} e^{2} / \hbar v$ is the Sommerfeld parameter, $\mathrm{Z}_{1}$ and $\mathrm{Z}_{2}$ the atomic number of interacting nuclei and $v$ their relative velocity. $S(E)$ is a smoothly varying function of energy because the gross of the Coulomb barrier penetration factor is compensated for owing to the presence of the inverse of the Gamow factor. In the case of resonant reactions, proceeding through an excited state of the intermediate compound nucleus, significant deviations from the smooth behaviour might be expected.

Therefore, extrapolation can introduce large systematic errors since the occurrence of unknown or unpredicted resonances can dramatically enhance the astrophysical $S(E)$-factor, strongly influencing astrophysical models. These considerations apply to both low-energy resonances and sub-threshold resonances as they may produce sizeable modifications of the $\mathrm{S}$-factor due to, for instance, interference with another resonance, especially in the case of broad resonances $[1,2]$.

Great improvements in the experimental approach used to measure vanishingly small cross sections have been devised, making it possible to extend the measurements of some reactions down to astrophysical energies (see, for instance, [3]). However, approaching interaction energies comparable with the electron binding energies in atoms the presence of atomic electrons cannot be neglected. Electron clouds lead to an enhancement of the astrophysical factor related to the shielding of the nuclear charges by the surrounding negatively-charged electrons and not to nuclear interaction (see $[1,2]$ for a general discussion and [4] and references therein for and example study). Even in those few cases when the reaction cross sections were measured inside the Gamow window, electron screening prevented the access to the bare-nucleus cross section, making extrapolation unavoidable.

Indeed, electron screening behaves differently in the laboratory and in astrophysical environments, as in the former projectile and target are in the form of ions and atoms or molecules, while in stars, for instance, matter is in the form of plasma. However, our present understanding of the electron screening effect is rather imperfect as experimental values often exceed theoretical upper limits [5], thus potential systematic errors might be introduced in the evaluation of the bare-nucleus $S(E)$ and, as a consequence, in its extrapolation to low energies.

Because of the problems affecting direct measurements at astrophysical energies, indirect techniques have been developed to bypass them and attain the astrophysical factor at low energies. For instance, the Trojan Horse Method (THM) [6] is a valid technique to get information on the $\mathrm{S}(\mathrm{E})$ at astrophysical energies for reactions having charged particles and neutrons in the exit channel, with no Coulomb and centrifugal barrier suppression neither electron screening. In the case of radiative capture reactions, the Asymptotic Normalization 


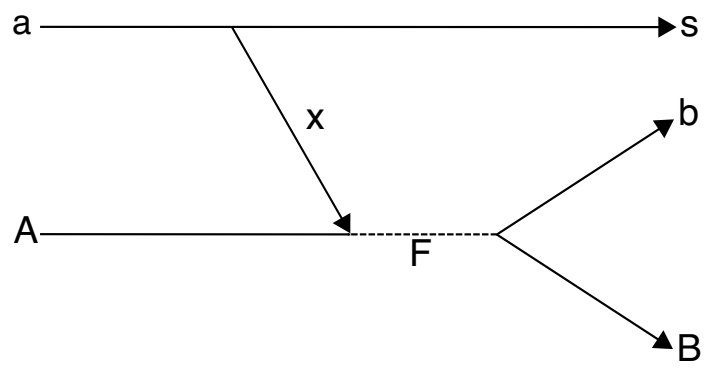

Figure 1. Sketch of the participant $(x)$-spectator $(s)$ mechanism leading to the population of excited states of the intermediate nucleus $F$, necessary condition for the application of the THM formalism.

Coefficient (ANC) [7] approach has allowed to obtain the direct-capture zero-energy S(E) factor with very high accuracy. The Coulomb dissociation (CD) was also developed [8] to determine the low-energy cross section of charged-particle induced reactions having a $\gamma$-ray in the exit channel.

\section{The THM for resonant reactions}

The THM has been established as a tool to investigate low-energy nuclear reactions by C. Spitaleri $[9,10]$ following the pioneering work of G. Baur [11]. In more recent years, A.M. Mukhamedzhanov has strongly improved the theoretical formalism [12, 13], introducing the modified R-matrix approach, allowing for the analysis of multi-resonance reactions with unprecedented accuracy (see, for instance, [14-17]). These upgrade in the theory has impacted astrophysics, as it is shown in the case of evolved star nucleosynthesis and presolar grains $[18,19]$. In the THM general framework, the cross section of the $A(x, b) B$ reaction is obtained through the $A(a, b B) s$ reaction performed at high energies (several tens of $\mathrm{MeV}$ ), so it is not hindered by the Coulomb and centrifugal barriers of the target-projectile system and no electron screening enhancement alters the trend of the cross section. However, particle $x$ is virtual so the $A(x, b) B$ THM is half-off-energy-shell (HOES) and cannot be simply compared with the corresponding direct (on-energy-shell, OES) cross section [12]. The modified $\mathrm{R}$-matrix approach has been introduced to extract the astrophysical S-factor of interest from the quasi-free $(\mathrm{QF})$ reaction yield, accounting for HOES effects.

Adopting the plane wave approximation (PWA) in the prior form and neglecting, for sake of simplicity, spins of the involved particles, the amplitude of the process $a+A \rightarrow b+B+s$ (Fig.1) takes the form:

$$
M^{P W A(p r i o r)}\left(P, \mathbf{k}_{a A}\right)=\left\langle\chi_{s F}^{(0)} \Psi_{b B}^{(-)}\left|V_{x A}\right| \varphi_{a} \varphi_{A} \chi_{a A}^{(0)}\right\rangle,
$$

where $P=\left(\mathbf{k}_{s F}, \mathbf{k}_{b B}\right)$ is the six-dimensional momentum describing the three-body system $s$, $b$ and $B \cdot \chi_{a A}^{(0)}=\exp \left(i \mathbf{k}_{a A} \cdot \mathbf{r}_{a A}\right), \chi_{s F}^{(0)}=\exp \left(i \mathbf{k}_{s F} \cdot \mathbf{r}_{s F}\right), \mathbf{r}_{i j}$ and $\mathbf{k}_{i j}$ are the relative coordinate and relative momentum of $\mathrm{i}$ and $\mathrm{j}$ nuclei, $\Psi_{b B}^{(-)}$is the wave function of the fragments $b$ and $B$ in the exit channel, $F=b+B, V_{x A}$ is the interaction potential of $x$ and the target nucleus $A, \varphi_{a}$ and $\varphi_{A}$ are the bound state wave function of nuclei $a$ and $A$, respectively. If we assume that resonant reaction mechanism is dominant in the explored energy region, taking into account spins of the interacting particles, considering only the s-wave bound state $a=s+x$ and neglecting the internal degrees of freedom of the transferred particle $x$, we get the prior PWA 
amplitude of the THM cross section in the form $[6,13]$ :

$$
\begin{array}{r}
M^{P W A(\text { prior })}\left(P, \mathbf{k}_{a A}\right)=(2 \pi)^{2} \sqrt{\frac{1}{\mu_{b B} k_{b B}}} \varphi_{a}\left(\mathbf{p}_{s x}\right) \\
\times \sum_{J_{F} M_{F} j^{\prime} l l^{\prime} m_{j^{\prime}} m_{l} m_{l^{\prime}} M_{x}} i^{l+l^{\prime}}\left\langle j m_{j} l m_{l} \mid J_{F} M_{F}\right\rangle\left\langle j^{\prime} m_{j^{\prime}} l^{\prime} m_{l^{\prime}} \mid J_{F} M_{F}\right\rangle \\
\times\left\langle J_{x} M_{x} J_{A} M_{A} \mid j^{\prime} m_{j^{\prime}}\right\rangle\left\langle J_{s} M_{s} J_{x} M_{x} \mid J_{a} M_{a}\right\rangle \\
\times \exp \left[-i \delta_{b B l}^{h s}\right] Y_{l m_{l}}\left(-\hat{\mathbf{k}}_{b B}\right) \\
\times \sum_{v \tau=1}^{N}\left[\Gamma_{v b B j l J_{F}}\right]^{1 / 2}\left[\mathbf{A}^{-1}\right]_{v \tau} Y_{l^{\prime} m^{\prime}}^{*}\left(\hat{\mathbf{p}}_{x A}\right) \\
\times \sqrt{\frac{R_{x A}}{\mu_{x A}}}\left[\Gamma_{v x A l^{\prime} j^{\prime} J_{F}}\left(E_{x A}\right)\right]^{1 / 2} P_{l^{\prime}}^{-1 / 2}\left(k_{x A}, R_{x A}\right) \\
\times\left(j_{l^{\prime}}\left(p_{x A} R_{x A}\right)\left[\left(B_{x A} l^{\prime}\left(k_{x A}, R_{x A}\right)-1\right)-D_{x A}\left(p_{x A}, R_{x A}\right)\right]\right. \\
\left.+2 Z_{x} Z_{A} e^{2} \mu_{x A} \int_{R_{x A}}^{\infty} \mathrm{d} r_{x A} \frac{O_{l^{\prime}}\left(k_{x A}, r_{x A}\right)}{O_{l^{\prime}}\left(k_{x A}, R_{x A}\right)} j_{l^{\prime}}\left(p_{x A} r_{x A}\right)\right) .
\end{array}
$$

Here, $\mathbf{p}_{i j}$ is the $i-j$ relative momentum in the case of off-energy-shell particles, thus $E_{i j} \neq$ $p_{i j}^{2} / 2 \mu_{i j}$ (while $\mathbf{k}_{i j}$ is calculated assuming the particles on-shell), $\delta_{b B l}^{h s}$ is the solid sphere scattering phase shift, $R_{x A}$ the $x+A$ channel radius,

$$
B_{x A} l^{\prime}\left(k_{x A}, R_{x A}\right)=R_{x A} \frac{\left.\frac{\partial O_{l^{\prime}}\left(k_{x A}, R_{x A}\right)}{\partial r_{x A}}\right|_{x_{x A}=R_{x A}}}{O_{l^{\prime}}\left(k_{x A}, R_{x A}\right)}
$$

is the logarithmic derivative as in the R-matrix method,

$$
O_{l^{\prime}}\left(k_{x A}, R_{x A}\right)=\sqrt{\frac{k_{x A} R_{x A}}{P_{l^{\prime}}\left(k_{x A}, R_{x A}\right)}} \exp \left[-i \delta_{x A}^{h s}\right]
$$

is the outgoing spherical wave, $P_{l^{\prime}}\left(k_{x A}, R_{x A}\right)$ the $l^{\prime}$-wave penetrability factor,

$$
D_{x A} l^{\prime}\left(p_{x A}, R_{x A}\right)=R_{x A} \frac{\left.\frac{\partial j_{l^{\prime}}\left(p_{x A}, R_{x A}\right)}{\partial r_{x A}}\right|_{r_{x A}=R_{x A}}}{j l^{\prime}\left(p_{x A}, R_{x A}\right)}
$$

the logarithmic derivative and $j_{l^{\prime}}\left(p_{x A}, R_{x A}\right)$ the spherical Bessel function, $N$ the number of the levels included. This is a generalization of the R-matrix approach because we consider reactions with three particles in the exit channel, where the TH-nucleus $a$ in the initial states carries the transferred particle $x$, which is off-energy-shell.

Eq.3 has four important consequences:

- $\mathbf{A}_{v \tau}$ is the same level matrix as in the conventional R-matrix theory [20]. Therefore, it depends on the entry and exit channels reduced width amplitudes $\gamma$, energy levels and energy shifts. All of them can be extracted by fitting the experimental THM cross section and then can be used to deduce the $A(x, b) B$ astrophysical factor. In this way, we have an exact parameterisation of the astrophysical factor with no need of extrapolation. HOES effects can affect the phases determining interference and the relative heights of the resonances, but the reduced widths $\gamma$, containing the nuclear structure effects, appear in the same way in THM and direct data. 
- The presence of the factor $P_{l^{\prime}}^{-1 / 2}\left(k_{x A}, R_{x A}\right)$ eliminates the penetration of Coulomb and centrifugal barriers in the $x+A$ channel, which is the entry channel of the reaction of astrophysical importance. The compensation of this penetrability factor is the main advantage of the THM, since it allows one to measure the astrophysical factor of the reaction of astrophysical interest down to zero energy. Moreover, resonances that can be populated with large $l$ only are not suppressed by the centrifugal barrier, thus they can be observed even in those cases they are very weak in direct measurements, making the THM a powerful spectroscopic tool.

- The use of the PWA implies that normalisation is accomplished by extending the indirect measurement to an energy region where directly measured data are available and scaling the deduced $\gamma$-widths to match the values in the literature. This is because in PWA absolute values are greatly overestimated. However, Eq. 3 can be modified as the $a-A$ and the $s-F$ interactions can be treated within the more advanced distorted waves (DWBA) and the CDCC formalisms [13]. This is a very important point as it opens the possibility to make unnecessary normalisation to direct data, at present a major drawback of THM. This is especially important in the investigation of reactions induced by radioactive ion beams, where direct data might be absent or scarce.

- In a THM experiments three particles are emitted in the exit channel. From the measurement of the energies and the angles of emissions of two out of three emitted particles, all the kinematic variables can be calculated. The most important parameter for astrophysical applications is the $x-A$ relative energy. Following [6], under the non essential hypothesis that the nucleus $a$ undergoing breakup is at rest in the laboratory system (similar formula can be found for breakup of the projectile), the $x-A$ relative energy can be written as:

$$
E_{x-A}=\frac{m_{x}}{m_{x}+m_{A}} E_{A}-\frac{p_{s}^{2}}{2 \mu_{s F}}+\frac{\mathbf{p}_{s} \cdot \mathbf{p}_{A}}{m_{x}+m_{A}}-\varepsilon_{s x},
$$

where $m_{i}, \mathbf{p}_{i}$ and $E_{i}$ are the mass, momentum and energy of the i-th particle, $\mu_{s F}$ the $s-F$ reduced mass and $\varepsilon_{s x}$ the $x-s$ binding energy. Part of the projectile energy is spent to break the impinging nucleus $a$ and thanks to the $x-s$ inter cluster motion, astrophysical energies can be achieved in the $x-A$ channel of the TH reaction using beam energies of few tens of $\mathrm{MeV}$, bypassing Coulomb barrier. Moreover, since high beam energies are used, the electron screening enhancement does not occur. Furthermore, negative $E_{x-A}$ energies can be explored by choosing a suitable combination of beam energy, spectator momentum and target nucleus $a$. From the THM measurement of sub-threshold states, yielding the reduced widths $\gamma$, the ANC can be deduced, making it possible to connect the two indirect approaches [21-23].

In the past years, a number of studies have been accomplished to validate the THM approach, making the method very robust. For instance, the effect of momentum distribution variations on the deduced astrophysical factors was considered. The use of realistic distributions in the place of the simple Hulthén function in momentum space (in the case of deuteron) has been investigated in [24], while the influence of the experimental momentum distribution in the place of the theoretical one was evaluated in [25, 26]. In both cases, changes comparable or lower then the statistical uncertainty were retrieved, since events in a momentum region of particle $s$ below about $40 \mathrm{MeV} / \mathrm{c}$ are usually considered in the data analysis [27]. The use of DWBA in the place of the PWA were tested in [28], showing again negligible changes in the deduced astrophysical factors, if the momentum window is kept within the Shapiro limit [27]. The method has been extended to measurements of neutron induced reactions [29] and to reactions involving radioactive nuclei [30-32]. 


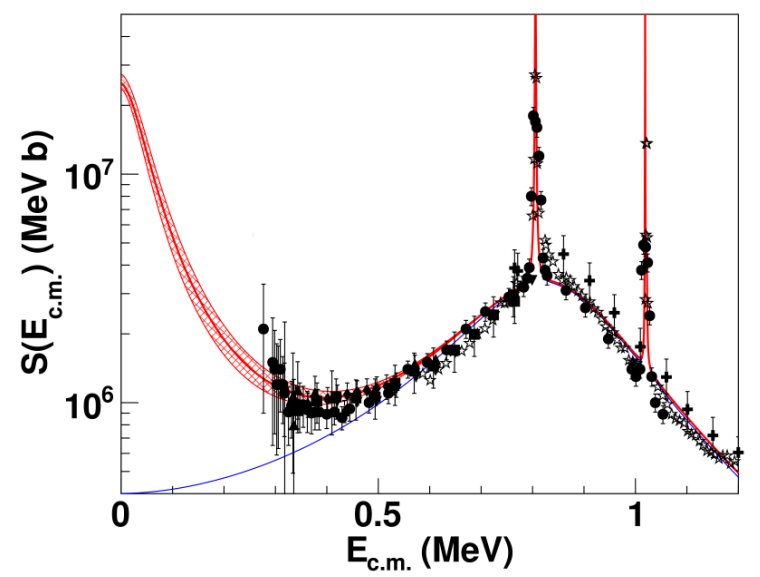

Figure 2. R-matrix calculated $\mathrm{S}(\mathrm{E})$-factor of the ${ }^{13} \mathrm{C}(\alpha, n){ }^{16} \mathrm{O}$ reaction (red band) from Ref. [22]. The $\mathrm{R}$-matrix $\mathrm{S}(\mathrm{E})$-factor not including the threshold resonance is displayed by the blue line. Black symbols are used for direct data normalized as in [37]. Different marks are used for each data set: diamonds for [38], crosses for [39], downward triangles for [40], squared for [41], circles for [36], stars for [42] and upward triangles for [37].

\section{The sub-threshold resonance case: the ${ }^{13} \mathrm{C}(\alpha, n){ }^{16} \mathrm{O}$ reaction}

In a class of evolved stars, those belonging to the so-called asymptotic giant branch (AGB), very peculiar conditions are present making it possible to synthesise nuclei heavier than iron through a succession of slow neutron captures, followed by decay of the formed unstable nuclei. This path to heavy nuclei is named s-process and it is responsible of the production of $\sim 50 \%$ of nuclei with $\mathrm{A} \approx 56$. In AGB stars, protons from the outer layers are mixed downward and quickly captured by carbon nuclei, eventually leading to the formation of a ${ }^{13} \mathrm{C}$ pocket [34]. Then, ${ }^{13} \mathrm{C}$ nuclei emits neutrons through the ${ }^{13} \mathrm{C}(\alpha, n){ }^{16} \mathrm{O}$ reaction, at temperatures varying between $0.8 \times 10^{8} \mathrm{~K}$ and $1 \times 10^{8} \mathrm{~K}$ [35], which can be captured by seed nuclei to build up heavier nuclei, later transported to the stellar surface.

At $0.910^{8} \mathrm{~K}$, the energy range where the ${ }^{13} \mathrm{C}(\alpha, n){ }^{16} \mathrm{O}$ reaction is most effective, the Gamow window [2], is $\sim 140-230 \mathrm{keV}$. In such region, its direct measurement is very challenging because of the Coulomb barrier, exponentially suppressing the cross section. On the other hand, also extrapolation is very complicated due to the presence of a threshold resonance determined by the population of the $6.356 \mathrm{MeV}$ level in ${ }^{17} \mathrm{O}$. Indeed, at $\sim 300 \mathrm{keV}$ the cross section of the ${ }^{13} \mathrm{C}(\alpha, n){ }^{16} \mathrm{O}$ reaction is already as low as $\sim 10^{-10} \mathrm{~b}$, thus direct measurements stopped at $\sim 280 \mathrm{keV}$ [36]. Moreover, direct measurements show contradicting results also at $\mathrm{MeV}$ energies, owing to uncertainties in the absolute normalisation. Therefore, extrapolation is necessary at present to assess the astrophysical factor of the ${ }^{13} \mathrm{C}(\alpha, n){ }^{16} \mathrm{O}$ reaction at astrophysical energy, but large errors might be introduced owing to the systematic uncertainty affecting high energy data. Therefore, [37] employed a broad data set including renormalised ${ }^{13} \mathrm{C}(\alpha, n){ }^{16} \mathrm{O}$ astrophysical factors to perform an extensive R-matrix fit; a $100 \mathrm{keV}$ astrophysical factor $\mathrm{S}(100 \mathrm{keV})=3.3_{-1.4}^{+1.8} \times 10^{6} \mathrm{MeVb}$ was then obtained. The renormalised data-set is shown in Fig. 2 as black symbols.

The THM S-factor is shown instead as a red band in Fig.2. Thanks to our approach, a very accurate result is obtained with no need of extrapolation down to zero energy, making it 


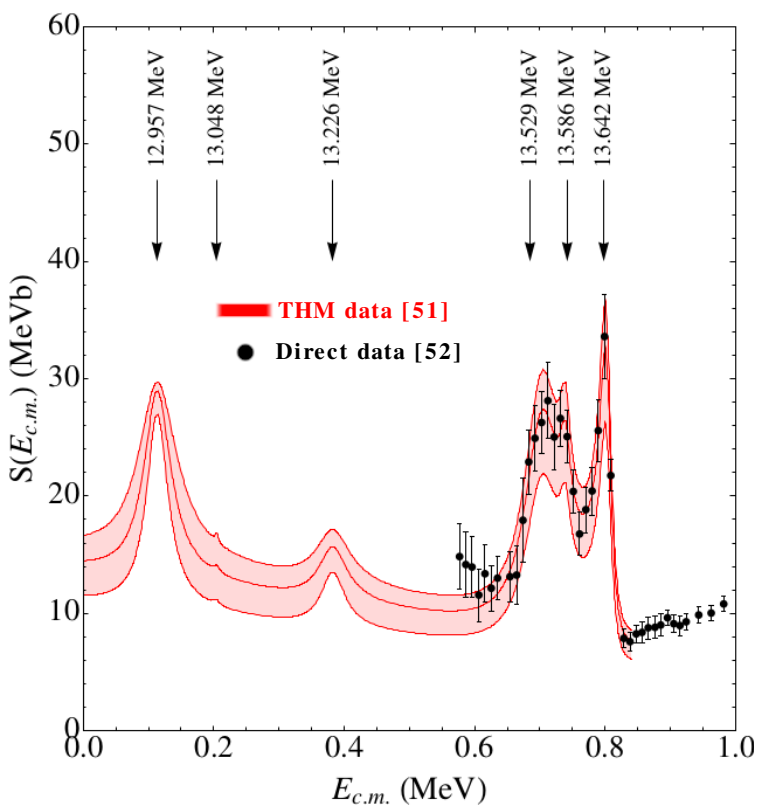

Figure 3. THM astrophysical factor of the ${ }^{19} \mathrm{~F}\left(p, \alpha_{0}\right){ }^{16} \mathrm{O}$ reaction, normalized to the data from [52] above $600 \mathrm{keV}$, as shown in [51]. The middle red line marks the recommended S-factor, while the upper and lower red lines stand for upper and lower limits set by combined statistical, normalization and energy shift error. The solid symbols represent the direct astrophysical factor in [52]. Finally, the arrows mark the ${ }^{20} \mathrm{Ne}$ states contributing to the $\mathrm{S}(\mathrm{E})$-factor.

possible to supply a very accurate reaction rate for astrophysical modelling. This is possible since we could directly access the $6.356 \mathrm{MeV}$ level in ${ }^{17} \mathrm{O}$, sitting at $-3 \mathrm{keV}$ in the ${ }^{13} \mathrm{C}-\alpha$ relative energy spectrum. The recommended THM S-factor at $\mathrm{E}_{c . m} .=100 \mathrm{keV}$ is $5.3 \pm$ $0.9 \times 10^{6} \mathrm{MeVb}$ [21, 22, 45], about $40 \%$ larger than the value provided by [37]. The most important result is a significant reduction of the uncertainty on the ${ }^{13} \mathrm{C}(\alpha, n){ }^{16} \mathrm{O}$ S-factor at the Gamow peak, which decreased from about $50 \%$ to about $20 \%$. Recently, two works $[43,44]$ have determined new interesting spectroscopic information about the $6.356 \mathrm{MeV}$. The consequences on the ${ }^{13} \mathrm{C}(\alpha, n){ }^{16} \mathrm{O}$ S-factor are presently under investigation $[45,46]$.

\section{Resonances above the threshold: the ${ }^{19} \mathrm{~F}\left(p, \alpha_{0}\right){ }^{16} \mathrm{O}$ reaction}

Since the s-process is quite complicated, involving large nuclear physics input as well as peculiar astrophysical conditions, the possibility to constrain the physical parameters (such as density and temperature) characterizing the stellar interior is very appealing. Fluorine might represent a strong constraint of stellar internal structure since its abundance is very sensitive to the physical conditions in the inner layers of AGB stars [47]. This entails the understanding of fluorine nucleosynthesis, which is incomplete to date. A possible explanation is the poor accuracy of our present picture of the fluorine destruction due to extra-mixing processes [47], where fluorine is exposed to protons at temperatures $\lesssim 4 \times 10^{7} \mathrm{~K}$.

The ${ }^{19} \mathrm{~F}(p, \alpha){ }^{16} \mathrm{O}$ reaction is the main destruction channel of fluorine in this scenario. However, only one set of direct data is available so far at the energies where fluorine burning is most effective $\left(\mathrm{E}_{c . m} . \leq 300 \mathrm{keV}\right.$, the Gamow energy [2]), still with quite large errors 
[53]. Furthermore, only the $\alpha_{0}$ channel, corresponding to the emission of $\alpha$-particles off ${ }^{20} \mathrm{Ne}$ compound system leaving ${ }^{16} \mathrm{O}$ in its ground state, has been investigated, being considered the larger contributor to the total cross section [53]. Before, only extrapolations were available [49] below about $500 \mathrm{keV}$, showing a non resonant behaviour, sharply contradicting the trend of the astrophysical factor at higher energies. This very simple recommended extrapolation to astrophysical energies has triggered the reassessment of the nuclear reaction rates involved in fluorine production and destruction, in particular by using the THM in its version modified to deal with resonant reactions [50, 51].

To this purpose, the $\mathrm{QF}{ }^{2} \mathrm{H}\left({ }^{19} \mathrm{~F}, \alpha^{16} \mathrm{O}\right) n$ reaction at $50 \mathrm{MeV}$ beam energy was measured by means of a ${ }^{19} \mathrm{~F}$ beam impinging onto deuterated polyethylene targets, thus using deuterons to transfer protons and induce the ${ }^{19} \mathrm{~F}(p, \alpha){ }^{16} \mathrm{O} \mathrm{QF}$ reaction. More details on the experiment are given in [50]. Here we underscore that by using Eq.3 we derived the reduced widths of a number of resonances and, in particular, of a $113 \mathrm{keV}$ peak sitting right inside the Gamow window, which might have important consequences for astrophysics. The $p-{ }^{19} \mathrm{~F}$ relative energy spectrum spanned an energy interval from 0 to about $1 \mathrm{MeV}$, making it possible to normalise the THM astrophysical factor to the existing direct data. In the original work [50], THM data were normalised to a weighed average of direct data in the energy window $0.6-0.8 \mathrm{MeV}$ [49]. Later, new direct data were made available in the normalisation energy region [52], leading to a reanalysis of the THM data [51], and at astrophysical energies [53]. A new THM experiment was carried out, improving statistics at astrophysical energies in the case of the $\alpha_{0}$ channel, and also aiming at exploring the $\alpha_{1}$ channel [54].

Fig. 3 shows the $S(E)$-factor calculated with the resonance parameters from the fitting of THM data below $600 \mathrm{keV}$. The middle red curve marks the S(E)-factor computed using the parameters from the best fit, while the red band arises from the uncertainties on the resonance parameters, due to the combined statistical and systematic error. An average error of $20 \%$ is obtained. At present, the main source of uncertainty is due to the non resonant contribution to the astrophysical factor, since the one given in [49] is based on a very simple calculation. New direct measurements are of utmost importance to have a more realistic non-resonant contribution at low energies.

\section{References}

[1] Rolfs C E and Rodney W S 1998 Cauldrons in the cosmos (Chicago: University of Chicago Press)

[2] Iliadis C 2007 Nuclear Physics of Stars (New York: Wiley)

[3] Best A et al. 2016 Eur. Phys. J. A 5272

[4] La Cognata M, Spitaleri C, Tumino A et al. 2005 Phys. Rev. C 72065802

[5] Strieder F, Rolfs C, Spitaleri C and Corvisiero P . 2001 Naturwissenschaften 88461

[6] Tribble R E, Bertulani CA, La Cognata M et al. 2014 Reports on Progress in Physics 77 106901

[7] Mukhamedzhanov A M, Clark HL, Gagliardi CA et al. 1997 Phys. Rev. C 561302

[8] Baur G and Rebel H 1994 Journal of Physics G Nuclear Physics 201

[9] Spitaleri C, Mukhamedzhanov A M, Blokhintsev, L. D et al. 2011 Physics of Atomic Nuclei 741725

[10] Spitaleri C 1991 Proceedings of the 5Th Winter School on Hadronic Physics: Problems of Fundamental Modern Physics II (Singapore: World Scientific) 21

[11] Baur G 1986 Physics Letters B 178135

[12] Mukhamedzhanov A M, Blokhintsev LD, Irgaziev BF et al. 2008 Journal of Physics G Nuclear Physics 35014016 
[13] Mukhamedzhanov A M 2011 Phys. Rev. C 84044616

[14] La Cognata M, Romano S, Spitaleri C et al. 2006 European Physical Journal A 27249

[15] La Cognata M, Spitaleri C, Tribble R E et al. 2008 Journal of Physics G Nuclear Physics 35014014

[16] La Cognata M, Goldberg VZ, Mukhamedzhanov AM et al. 2009 Phys. Rev. C 80012801

[17] La Cognata M , Spitaleri C, Mukhamedzhanov A M et al. 2010 The Astrophysical Journal 7231512

[18] Palmerini S, La Cognata M, Cristallo S. et al. 2011 The Astrophysical Journal 7293

[19] Palmerini S, Sergi, M. L., La Cognata, M et al. 2013 The Astrophysical Journal 764128

[20] Lane A M and Thomas R G 1958 Reviews of Modern Physics 30257

[21] La Cognata M, Spitaleri C, Trippella O et al. 2012 Physical Review Letters 109232701

[22] La Cognata M, Spitaleri C, Trippella O et al. 2013 The Astrophysical Journal 777143

[23] La Cognata M , Spitaleri C, Cherubini S et al. 2016 Acta Physics Polonica B 47681

[24] Lamia L, La Cognata M, Spitaleri C et al. 2012 Phys. Rev. C 85025805

[25] Pizzone R G, Spitaleri C, Cherubini S et al. 2005 Phys. Rev. C 71058801

[26] Pizzone RG, Spitaleri C, Bertulani CA et al. (2019) Phys. Rev. C 87025805

[27] Spitaleri C, La Cognata M, Lamia L et al. 2016 European Physical Journal A 5277

[28] La Cognata M, Spitaleri C, Mukhamedzhanov A. et al. 2010 Nucl. Phys. A 834 658C

[29] Gulino M, Spitaleri C, Tang X D et al. 2013 Phys. Rev. C 87012801

[30] Cherubini C, Gulino M, Spitaleri C et al. 2015 Phys. Rev. C 92015805

[31] Pizzone R G, Roeder B T, McCleskey M et al. 2016 European Physical Journal A 52 24

[32] La Cognata M, Pizzone RG, Jose J et al. 2017 The Astrophysical Journal 84665

[33] Heil M, Detwiler R, Azuma R E et al. 2008 Phys. Rev. C 78025803

[34] Gallino R, Arlandini C, Busso M; et al. 1998 Astrophys. J. 497388

[35] Busso M, Gallino R, Wasserburg G J et al. 1999 Annual Review of Astronomy and Astrophysics 37239

[36] Drotleff H W, Denker H, Knee H et al. 1993 Astrophys. J. 414735

[37] Heil M, Kaeppeler F, Uberseder E et al. 2008 Phys. Rev. C 78025803

[38] Davids C 1968 Nucl. Phys. A110 619

[39] Bair J and Haas F 1973 Phys. Rev. C 71356

[40] Kellogg S, Vogelaar R and Kavanagh RW 1989 Bull. Am. Phys. Soc. 341192

[41] Brune CR, Licot I and Kavanagh RW 1993 Phys. Rev. C 483119

[42] Harissopulos S, Becker HW, Hammer JW et al. 2005 Phys. Rev. C 72 062801(R)

[43] Avila M L, Rogachev G V, Koshchiy E et al. 2015 Phys. Rev. C 91048801

[44] Faestermann T, Mohr P, Hertenberger R et al. 2015 Phys. Rev. C 92052802

[45] Trippella O and La Cognata M 2017 Astrophys. J. 83741

[46] Cristallo S, La Cognata M, Massimi C et al. 2018 Astrophys. J. 859105

[47] Lugaro M, Ugalde C, Karakas AI et al. 2004 Astrophys. J. 615934

[48] Lombardo I, Dell'Aquila D, Di Leva A et al. 2015 Phys. Lett. B 748178

[49] Angulo C, Arnould M, Rayet M et al. 1999 Nuclear Physics A 6563

[50] La Cognata M, Mukhamedzhanov A M, Spitaleri C et al. 2011 The Astrophysical Journal Letters 739 L54

[51] La Cognata M, Palmerini S, Spitaleri C et al. 2015 The Astrophysical Journal 805128

[52] Lombardo I, Dell'Aquila D, Campajola L et al. 2013 Journal of Physics G Nuclear Physics 40125102 
[53] Lombardo I et al. 2015 Phys. Lett. B 748178

[54] Indelicato I, La Cognata M, Spitaleri C et al. 2017 The Astrophysical Journal 84519 